\title{
Exact solutions of the aspherical axisymmetric gravo-magnetic condensation
}

\author{
P. Hennebelle` \\ Laboratoire de radioastronomie millimétrique, UMR 8540 du CNRS, École normale supérieure et Observatoire \\ de Paris, 24 rue Lhomond, 75231 Paris Cedex 05, France \\ Department of Physics and Astronomy, Cardiff University, PO Box 913, 5 The Parade, Cardiff CF24 3YB, \\ Wales, UK \\ Received 27 February 2001 / Accepted 8 August 2001

\begin{abstract}
We find exact and explicit self-similar solutions of the axisymmetric MHD equations of a self-gravitating polytropic gas. These solutions are able to describe a flat (uniform density) subsonic internal core, contracting homologously out of a collapsing cloud with aspherical pressure, aspherical gravitational potential, magnetic field and rotation. Two of the solutions describing the collapse of a rotating and magnetized cloud present outflow at the pole or at the equator.
\end{abstract}

Key words. accretion, accretion disks - gravitation - hydrodynamics - magnetohydrodynamics (MHD) ISM: clouds

\section{Introduction}

\subsection{Previous work}

The gravitational contraction of a cloud has been extensively studied numerically and analytically in the context of star formation and molecular cloud core collapse. Most of the analytical studies assume a polytropic equation of state: $D / D t\left(P \rho^{-\gamma}\right)=0$, a simple, spherical, cylindrical or disk-like geometry, and look for exact or approximate solutions using various analytical techniques (e.g. self-similarity).

\subsubsection{Hydrodynamical collapse}

Most of the available work adresses the problem of hydrodynamical spherical collapse (Penston 1966, 1969a, 1969b; Larson 1969; Shu 1977; Hunter 1977; Goldreich \& Weber 1980; Yahil 1983; Munier \& Feix 1983; Bouquet et al. 1985; Whitworth \& Summers 1985) using self-similar approaches.

Larson (1969) and Penston (1969) first reduced the fluid equations of a self-gravitating isothermal gas into ordinary differential equations of the radius, $r$ only. They also derived an exact solution, that presents uniform density and homologous velocity in the internal part of the cloud (see Fig. 1). At some point, the velocity (relative to the similarity profile) reaches the sound speed and a bifurcation occurs, the density decreases and the solution tends asymptotically to the singular isothermal sphere. This

\footnotetext{
* e-mail: patrick.hennebelle@ens.fr
}

result has been demonstrated analytically by Whitworth \& Summers (1985) in the isothermal case $(\gamma=1)$ and by Bouquet et al. (1985) for any value of $\gamma$. Whitworth \& Summers find that the central density is quantised, i.e. there are bands of central density for which the solution does not go through the sonic surface. They claim that the Larson-Penston solution, that belongs to the first allowed band, represents the subsonic core of a collapsing cloud and is able to describe better the gravitational collapse than other solutions found by Hunter (1977) and Shu (1977) that belong to the other bands and that are much more centrally peaked.

These studies leave aside important aspects such as rotation or the magnetic field (Mouschovias 1978).

\subsubsection{MHD collapse}

Some more recent works investigate the collapse of a magnetized gas, making various approximations. Galli \& Shu (1993) use a self-similar approach and develop a perturbative solution, Nakamura et al. (1999), Contopoulos et al. (1998) and Basu (1997) consider the thin disk approximation, Chiueh \& Chou (1994) and Li (1998a, 1998b) neglect the magnetic tension and assume an isotropic magnetic support. A notable exception is the work of Low (1992) who was able, using a self-similar analysis, to find exact solutions of the collapse of the $\gamma=4 / 3$ magnetized polytrope. Low reduces the MHD equations to the static equations modified by a radial static force, that is to say, the Low solutions are static in the similarity space. An important restriction of his work is that there is an equilibrium 
between the non radial components of the Lorentz, the gravitational and the pressure forces and consequently the orthoradial and azimuthal components of the velocity field vanish. Thus, as far as the author knows, and in spite of the importance of considering these processes together, there are no exact solutions in the literature describing a gravitational collapse of a magnetized and rotating cloud.

\subsection{Aim of the paper}

In this paper, we find exact and explicit solutions describing the gravitational collapse or expansion of selfgravitating polytropic gas of uniform density including magnetic field, rotation, aspherical thermal pressure and aspherical gravitational potential. The main difficulty and originality of the present study is that the various fields have a non-trivial angular dependence.

The derivation of exact solutions is always of great interest; they give explicit examples of how the different terms counterbalance and can constitute the starting point of further analytical studies like stability or bifurcation (Goldreich \& Weber 1980; Bouquet et al. 1985; Blottiau et al. 1988; Ringeval \& Bouquet 2000). Such solutions can also be used as benchmarks, crucial in the testing of the complex MHD codes developed for studies related to the gravitational collapse. Indeed, the importance of code testing cannot be overemphasized.

In Sect. 2, we apply the dynamical rescaling method introduced by Munier \& Feix (1983), to the MHD equation of a self-gravitating polytropic gas. Considering axisymmetric geometry, density independent of $r$ and homologous velocity fields, we first reduce the system into a set of 8 equations that depend on $\widehat{t}$, the rescaled time, and $\theta$ the colatitude only and finally reduce it into 7 ordinary differential equations of $\theta$.

In Sect. 3, we restrict the problem further considering a uniform density field and homologous velocity. We find various exact solutions of the stationary (with respect to the rescaled time, $\widehat{t}$ ) hydrodynamical and MHD equations. Some of the solutions with rotation and magnetic field have radial velocity fields that become positive (outflow) at the pole or at the equator. Section 4 presents various solutions, able to describe a collapse, with an anisotropic thermal pressure. Depending on $\gamma$, the polytropic index, some of the solutions present outflows at the pole or at the equator. Section 5 concludes the paper.

\section{Reduction of the system}

\subsection{The equations}

We consider the ideal MHD equations, in spherical coordinates, of a polytropic self-gravitating gas. We thus assume that the magnetic field is perfectly coupled to the gas, i.e. we do not take into account ambipolar diffusion. In the usual notation, we have:

$\partial_{t} \rho+\operatorname{div}(\rho \boldsymbol{V})=0$ $\rho\left(\partial_{t} \boldsymbol{V}+\boldsymbol{V} \cdot \operatorname{grad} \mathbf{V}\right)=$

$$
-\operatorname{grad} P+\rho \operatorname{grad} \Phi+\frac{1}{\mu_{0}} \operatorname{rot} \boldsymbol{B} \wedge \boldsymbol{B}
$$

$\partial_{t}\left(P \rho^{-\gamma}\right)+\boldsymbol{V} \cdot \operatorname{grad}\left(P \rho^{-\gamma}\right)=0$

$\Delta \Phi=-4 \pi G \rho$

$\operatorname{div} \boldsymbol{B}=0$

$\partial_{t} \boldsymbol{B}+\operatorname{rot}(\boldsymbol{V} \wedge \boldsymbol{B})=\mathbf{0}$.

\subsection{Magnetic field}

In the following, we consider axisymmetric geometries $\left(\partial_{\phi}=0\right)$ and use the divergence free form for the magnetic field (Low 1992):

$B_{r}=-\frac{1}{r^{2} \sin \theta} \partial_{\theta} H_{1}(t, r, \theta)$

$B_{\theta}=\frac{1}{r \sin \theta} \partial_{r} H_{1}(t, r, \theta)$

$B_{\phi}=\frac{1}{r^{2}} H_{2}(t, r, \theta)$

where $H_{2}$ has been introduced in order to have the same physical dimension as $H_{1}$.

Equation (5) is automatically satisfied and Eq. (6) reduces to the two equations:

$\partial_{t} H_{1}+V_{r} \partial_{r} H_{1}+\frac{1}{r} V_{\theta} \partial_{\theta} H_{1}=0$,

$\partial_{t} H_{2}+r \partial_{r}\left(\frac{1}{r \sin \theta} V_{\phi} \partial_{\theta} H_{1}+\frac{1}{r} V_{r} H_{2}\right)$
$+\frac{1}{r} \partial_{\theta}\left(V_{\theta} H_{2}-\frac{r}{\sin \theta} V_{\phi} \partial_{r} H_{1}\right)=0$.

\subsection{Dynamical rescaling}

The study of the system of Eqs. (1)-(6) is carried out using a method which generalizes the usual self-similar technique. Following Munier \& Feix (1983) and Bouquet et al. (1985), we perform the following transformation:

$\mathrm{d} t=\tau^{2}(t) \mathrm{d} \widehat{t}$

$r=a(t) \widehat{r}$

$P(t, r, \theta)=\pi(t) \widehat{P}(\widehat{t}, \widehat{r}, \theta)$,

$\rho(t, r, \theta)=d(t) \widehat{\rho}(\widehat{t}, \widehat{r}, \theta)$,

$\Phi(t, r, \theta)=f(t) \widehat{\Phi}(\widehat{t}, \widehat{r}, \theta)$,

$H_{1}(t, r, \theta)=h(t) \widehat{H}_{1}(\widehat{t}, \widehat{r}, \theta)$,

$H_{2}(t, r, \theta)=h(t) \widehat{H}_{2}(\widehat{t}, \widehat{r}, \theta)$. 
This procedure generalizes self-similarity since the transformed fields (e.g. $\widehat{\rho}$ ) are allowed to depend both on rescaled space and time whereas the self-similar solutions depend on rescaled space only. The time dependence of the new fields allows (at least numerically) the study of the convergence toward the self-similar solutions. This procedure also allows the study of the dynamical stability of the self-similar solution in the rescaled space.

The derivative against time and space are given by:

$\partial_{t}=\frac{1}{\tau^{2}} \partial_{\widehat{t}}-\frac{\dot{a}}{a} \widehat{r} \partial_{\widehat{r}}$

$\partial_{r}=\frac{1}{a} \partial_{\widehat{r}}$.

With:

$\begin{aligned} \widehat{V}_{r} & =\frac{\partial \widehat{r}}{\partial \widehat{t}} \\ \widehat{V}_{\theta} & =\widehat{r} \frac{\partial \theta}{\partial \widehat{t}} \\ \widehat{V}_{\phi} & =\widehat{r} \sin \theta \frac{\partial \phi}{\partial \widehat{t}}\end{aligned}$

we have:

$V_{r}=\frac{\partial r}{\partial t}=\frac{\partial a(t) \widehat{r}}{\partial t}=\dot{a}(t) \widehat{r}+\frac{a}{\tau^{2}} \widehat{V}_{r}$,

$V_{\theta}=r \frac{\partial \theta}{\partial t}=\frac{a}{\tau^{2}} \widehat{V}_{\theta}$,

$V_{\phi}=r \sin \theta \frac{\partial \phi}{\partial t}=\frac{a}{\tau^{2}} \widehat{V}_{\phi}$.

After some algebra, one finds that the system of Eqs. (1), (2), (3), (4), (10), (11) becomes in spherical coordinates (as the study of the angular dependence of the fields is our main goal, we write these equations explicitly):

$$
\begin{array}{r}
\partial_{\hat{t}} \widehat{\rho}+\frac{1}{\widehat{r}^{2}} \partial_{\widehat{r}}\left(\widehat{r}^{2} \widehat{V}_{r} \widehat{\rho}\right)+\frac{1}{\widehat{r} \sin \theta} \partial_{\theta}\left(\sin \theta \widehat{V}_{\theta} \widehat{\rho}\right)= \\
-\tau^{2}\left(\frac{\dot{d}}{d}+3 \frac{\dot{a}}{a}\right) \widehat{\rho},
\end{array}
$$

$$
\begin{aligned}
\partial_{\widehat{t}} \widehat{V}_{r} & +\widehat{V}_{r} \partial_{\widehat{r}} \widehat{V}_{r}+\frac{1}{\widehat{r}} \widehat{V}_{\theta} \partial_{\theta} \widehat{V}_{r}-\frac{\widehat{V}_{\theta}^{2}+\widehat{V}_{\phi}^{2}}{\widehat{r}} \\
& +2 \tau^{2}\left(\frac{\dot{a}}{a}-\frac{\dot{\tau}}{\tau}\right) \widehat{V}_{r}+\tau^{4} \frac{\ddot{a}}{a} \widehat{r}=-\frac{\pi \tau^{4}}{a^{2} d} \frac{\partial_{\widehat{r}} \widehat{P}}{\widehat{\rho}}+\frac{f \tau^{4}}{a^{2}} \partial_{r} \widehat{\Phi} \\
& -\frac{h^{2} \tau^{4}}{a^{6} d} \frac{1}{\widehat{\rho}} \frac{1}{\mu_{0} \widehat{r}^{2}}\left(\frac{1}{\widehat{r}} \widehat{H}_{2} \partial_{\widehat{r}}\left(\frac{\widehat{H}_{2}}{\widehat{r}}\right)+\frac{1}{\sin \theta} \partial_{\widehat{r}} \widehat{H}_{1}\right. \\
& \left.\times\left(\partial_{\widehat{r}}\left(\frac{1}{\sin \theta} \partial_{\widehat{r}} \widehat{H}_{1}\right)+\partial_{\theta}\left(\frac{1}{\widehat{r}^{2} \sin \theta} \partial_{\theta} \widehat{H}_{1}\right)\right)\right)
\end{aligned}
$$

$$
\begin{aligned}
\partial_{t} \widehat{V}_{\theta} & +\widehat{V}_{r} \partial_{\widehat{r}} \widehat{V}_{\theta}+\frac{1}{\widehat{r}} \widehat{V}_{\theta} \partial_{\theta} \widehat{V}_{\theta}+\frac{\widehat{V}_{r} \widehat{V}_{\theta}}{\widehat{r}}-\frac{\widehat{V}_{\phi}^{2} \cot \theta}{\widehat{r}} \\
& +2 \tau^{2}\left(\frac{\dot{a}}{a}-\frac{\dot{\tau}}{\tau}\right) \widehat{V}_{\theta}=-\frac{\pi \tau^{4}}{a^{2} d} \frac{1}{\widehat{r}} \frac{\partial_{\theta} \widehat{P}}{\widehat{\rho}}+\frac{f \tau^{4}}{a^{2}} \frac{1}{\widehat{r}} \partial_{\theta} \widehat{\Phi} \\
& -\frac{h^{2} \tau^{4}}{a^{6} d} \frac{1}{\hat{\rho}} \frac{1}{\mu_{0} \widehat{r}^{3} \sin \theta}\left(\widehat{H}_{2} \partial_{\theta}\left(\frac{\sin \theta}{\widehat{r}^{2}} \widehat{H}_{2}\right)\right. \\
& \left.+\partial_{\theta} \widehat{H}_{1}\left(\partial_{\widehat{r}}\left(\frac{1}{\sin \theta} \partial_{\widehat{r}} \widehat{H}_{1}\right)+\partial_{\theta}\left(\frac{1}{\widehat{r}^{2} \sin \theta} \partial_{\theta} \widehat{H}_{1}\right)\right)\right)
\end{aligned}
$$

$$
\begin{aligned}
& \partial_{\hat{t}} \widehat{V}_{\phi}+\widehat{V}_{r} \partial_{\widehat{r}} \widehat{V}_{\phi}+\frac{1}{\widehat{r}} \widehat{V}_{\theta} \partial_{\theta} \widehat{V}_{\phi}+\frac{\widehat{V}_{r} \widehat{V}_{\phi}}{\widehat{r}}+\frac{\widehat{V}_{\theta} \widehat{V}_{\phi} \cot \theta}{\widehat{r}} \\
& +2 \tau^{2}\left(\frac{\dot{a}}{a}-\frac{\dot{\tau}}{\tau}\right) \widehat{V}_{\phi}=\frac{h^{2} \tau^{4}}{a^{6} d} \frac{1}{\widehat{\rho}} \frac{1}{\mu_{0} \widehat{r}^{2} \sin \theta} \\
& \times\left(\frac{1}{\sin \theta} \partial_{\widehat{r}} \widehat{H}_{1} \partial_{\theta}\left(\frac{\sin \theta}{\widehat{r}^{2}} \widehat{H}_{2}\right)-\frac{1}{\widehat{r}} \partial_{\theta} \widehat{H}_{1} \partial_{\widehat{r}}\left(\frac{1}{\widehat{r}} \widehat{H}_{2}\right)\right), \\
& \partial_{\widehat{t}}\left(\widehat{P} \widehat{\rho}^{-\gamma}\right)+\widehat{V}_{r} \partial_{\widehat{r}}\left(\widehat{P} \widehat{\rho}^{-\gamma}\right)+\frac{1}{\widehat{r}} \widehat{V}_{\theta} \partial_{\theta}\left(\widehat{P} \widehat{\rho}^{-\gamma}\right)= \\
& -\tau^{2} \frac{d_{t}\left(\pi d^{-\gamma}\right)}{\pi d^{-\gamma}} \widehat{P} \widehat{\rho}^{-\gamma}, \\
& \frac{1}{\widehat{r}} \partial_{\widehat{r}^{2}}^{2}(\widehat{r} \widehat{\Phi})+\frac{1}{\widehat{r}^{2} \sin \theta} \partial_{\theta}\left(\sin \theta \partial_{\theta} \widehat{\Phi}\right)=-4 \pi G \frac{a^{2} d}{f} \widehat{\rho}, \\
& \partial_{\hat{t}} \widehat{H}_{1}+\widehat{V}_{r} \partial_{r} \widehat{H}_{1}+\frac{1}{\widehat{r}} \widehat{V}_{\theta} \partial_{\theta} \widehat{H}_{1}=-\tau^{2} \frac{\dot{h}}{h} \widehat{H}_{1}, \\
& \partial_{\hat{t}} \widehat{H}_{2}+\widehat{r} \partial_{\widehat{r}}\left(\frac{1}{\widehat{r} \sin \theta} \widehat{V}_{\phi} \partial_{\theta} \widehat{H}_{1}+\frac{1}{\widehat{r}} \widehat{V}_{r} \widehat{H}_{2}\right) \\
& +\frac{1}{\widehat{r}} \partial_{\theta}\left(\widehat{V}_{\theta} \widehat{H}_{2}-\frac{\widehat{r}}{\sin \theta} \widehat{V}_{\phi} \partial_{\widehat{r}} \widehat{H}_{1}\right)=-\tau^{2} \frac{\dot{h}}{h} \widehat{H}_{2} .
\end{aligned}
$$

We choose the scaling in Eqs. (12) in order to cancel explicit time dependencies in system of Eqs. (16)-(23). Thus we impose that:

$$
\left\{\begin{array}{l}
\tau^{2}(\dot{d} / d+3 \dot{a} / a)=K_{1}, \tau^{2}(\dot{a} / a-\dot{\tau} / \tau)=K_{2} \\
\tau^{4} \ddot{a} / a=K_{3}, \pi \tau^{4} /\left(a^{2} d\right)=K_{4} \\
f \tau^{4} / a^{2}=K_{5}, h^{2} \tau^{4} / a^{6} d=K_{6} \\
a^{2} d / f=K_{7}, \tau^{2}\left(d / d t\left(\pi d^{-\gamma}\right)\right) / \pi d^{-\gamma}=K_{8} \\
\tau^{2} \dot{h} / h=K_{9}
\end{array}\right.
$$

where $K_{i}$ are real numbers. Although there are 6 variables only and nine constraints, the system of Eqs. (24) admits the following solution:

$$
\left\{\begin{array}{l}
\tau(t)=\sqrt{\Omega_{0} \omega t+1}, a(t)=\left(\Omega_{0} \omega t+1\right)^{\lambda} \\
d(t)=\left(\Omega_{0} \omega t+1\right)^{-2}, \pi(t)=\left(\Omega_{0} \omega t+1\right)^{2 \lambda-4} \\
f(t)=\left(\Omega_{0} \omega t+1\right)^{2 \lambda-2}, h(t)=\left(\Omega_{0} \omega t+1\right)^{3 \lambda-2}
\end{array}\right.
$$

where $\omega$ and $\lambda$ are two real numbers, $\rho_{0}$ is the density at origin and the Jeans frequency: $\Omega_{0}=\left(4 \pi G \rho_{0}\right)^{1 / 2}$ has been introduced for later simplification.

With Eqs. (24), one obtains the dependence of the nine constants $K_{i}$ on $\omega$ and $\lambda$ :

$$
\left\{\begin{array}{l}
K_{1}=\Omega_{0} \omega(3 \lambda-2), K_{2}=\Omega_{0} \omega(\lambda-1 / 2), \\
K_{3}=\left(\Omega_{0} \omega\right)^{2} \lambda(\lambda-1), K_{4}=1, \\
K_{5}=1, K_{6}=1, K_{7}=1, \\
K_{8}=2 \Omega_{0} \omega(\lambda+\gamma-2), K_{9}=\Omega_{0} \omega(3 \lambda-2) .
\end{array}\right.
$$

With Eqs. (24) and (26), the system of Eqs. (16)-(23) is independent of the variable $t$. It can be seen that due to the transformation stated by Eqs. (12) new terms, that can be seen e.g. as source terms, appear in the new frame defined by the variable $\widehat{t}$ and $\widehat{r}$ (see e.g. Bouquet et al. 1985, for a discussion). 
If $\omega$ is positive, the transformation is defined for any value of $t$ and describes an expansion, and if $\omega$ is negative the density becomes infinite at $t=-1 /\left(\Omega_{0} \omega\right)$ and the transformation describes a collapse.

The transformation performed in this paper is slightly more general than the transformation performed by previous authors (Munier \& Feix 1983; Bouquet et al. 1985) who assume that $\pi(t)=d^{\gamma}(t)$, i.e. they impose that energy is conserved in the similarity space. This choice leads to $\lambda=2-\gamma$, whereas in the present study $\lambda$ is a free parameter, energy is not conserved in the similarity space since a source term appears in Eq. (20).

\subsection{Reduction into systems of ordinary differential equations}

In order to reduce further Eqs. (16)-(23), it is worthwhile to reduce these equations into systems of ordinary differential equations.

\subsubsection{Ordinary equations of the radius}

The first and obvious possible reduction is to consider fields independent of $\theta$. In this case, $V_{\theta}, V_{\phi}, H_{1}, H_{2}$ must vanish and with $\lambda=2-\gamma$, one obtains the following equations:

$\partial_{t} \widehat{\rho}+\frac{1}{\widehat{r}^{2}} \partial_{\widehat{r}}\left(\widehat{r}^{2} \widehat{V}_{r} \widehat{\rho}\right)=-\Omega_{0} \omega(4-3 \gamma) \widehat{\rho}$,

$$
\begin{aligned}
\partial_{\hat{t}} \widehat{V}_{r}+\widehat{V}_{r} \partial_{\widehat{r}} \widehat{V}_{r}+\Omega_{0} \omega(3-2 \gamma) \widehat{V}_{r} & +\left(\Omega_{0} \omega\right)^{2}(2-\gamma)(1-\gamma) \widehat{r} \\
& =-\frac{\partial_{\widehat{r}} \widehat{P}}{\widehat{\rho}}+\partial_{\widehat{r}} \widehat{\Phi}
\end{aligned}
$$

$\frac{1}{\widehat{r}} \partial_{\widehat{r}^{2}}^{2}(\widehat{r} \widehat{\Phi})=-4 \pi G \widehat{\rho}$

which is the system obtained by Yahil (1983) and by Bouquet et al. (1985). In the isothermal case $(\gamma=1)$ it reduces to the system obtained by Penston (1969) and Larson (1969) and extensively studied by Shu (1977), Hunter (1977) and Whitworth \& Summers (1985).

\subsubsection{Ordinary equations of the colatitude}

Another possibility is to reduce the system of Eqs. (16)(23), into a system that depends on the angle $\theta$ only.

This reduction is obtained by considering the fields:

$$
\left\{\begin{array}{l}
\widehat{t}=\widetilde{t} / \Omega_{0}, \widehat{V}_{r}(\widehat{t}, \widehat{r}, \theta)=\Omega_{0}(V(\widetilde{t}, \theta)-\lambda \omega) \widehat{r}, \\
\widehat{V}_{\theta}(\widehat{t}, \widehat{r}, \theta)=\Omega_{0} U(\widetilde{t}, \theta) \widehat{r}, \widehat{V} \phi(\widehat{t}, \widehat{r}, \theta)=\Omega_{0} W(\widetilde{t}, \theta) \widehat{r}, \\
\widehat{P}(\widehat{t}, \widehat{r}, \theta)=\rho_{0} \Omega_{0}^{2} \Pi(\widetilde{t}, \theta) \widehat{r}^{2}, \widehat{\rho}(\widehat{t}, \widehat{r}, \theta)=\rho_{0} R(\widetilde{t}, \theta), \\
\widehat{\Phi}(\widehat{t}, \widehat{r}, \theta)=\Omega_{0}^{2} \phi(\widetilde{t}, \theta) \widehat{r}^{2}, \\
\widehat{H}_{1}(\widehat{t}, \widehat{r}, \theta)=\sqrt{\mu_{0} \rho_{0} \Omega_{0} h_{1}(\widetilde{t}, \theta) \widehat{r}^{3}}, \\
\widehat{H}_{2}(\widehat{t}, \widehat{r}, \theta)=\sqrt{\mu_{0} \rho_{0}} \Omega_{0} h_{2}(\widetilde{t}, \theta) \widehat{r}^{3} .
\end{array}\right.
$$

With the definition of $V(\theta)$ (first equation), it is easily seen with Eqs. (15) and (25) that $V_{r}(r, \theta) \propto V(\theta)$. We also have $V_{\theta}(r, \theta) \propto U(\theta)$ and $V_{\phi}(r, \theta) \propto W(\theta)$.
As for the Larson-Penston solution, these fields diverge when $r$ goes to infinity and the solutions are valid in a finite domain only (until the velocity reaches the velocity of the sound or magneto-sonic waves).

Another important restriction is due to the fact that the magnetic field (see Eqs. (7), (8) and (9)) is proportional to the radius, $r$, and vanishes at the origin. Thus, the magnetic pressure compresses the gas and enhances the condensation process instead of supporting the gas against the gravitational collapse. However, in the following we will be able to add a uniform field and to avoid this restriction for some of the solutions (see Eqs. (58)-(59)).

With Eqs. (24), (26) and (30), the system of Eqs. (16)(23) becomes:

$\partial_{t} R+3 V R+\frac{1}{\sin \theta} \partial_{\theta}(\sin \theta U R)=2 \omega R$,

$\partial_{t} V+V^{2}+U \partial_{\theta} V-U^{2}-W^{2}-\omega V=-2 \frac{\Pi}{R}+2 \phi$

$$
-\frac{1}{R}\left(2 h_{2}^{2}+\frac{18}{\sin ^{2} \theta} h_{1}^{2}+\frac{3}{\sin \theta} h_{1} \partial_{\theta}\left(\frac{1}{\sin \theta} \partial_{\theta} h_{1}\right)\right),
$$

$\partial_{t} U+2 V U+U \partial_{\theta} U-W^{2} \cot \theta-\omega U=$

$$
\begin{array}{r}
-\frac{\partial_{\theta} \Pi}{R}+\partial_{\theta} \phi \\
-\frac{1}{R}\left(\frac{1}{\sin \theta} h_{2} \partial_{\theta}\left(\sin \theta h_{2}\right)+\frac{6}{\sin ^{2} \theta} \partial_{\theta} h_{1} h_{1}\right. \\
\left.+\frac{1}{\sin \theta} \partial_{\theta} h_{1} \partial_{\theta}\left(\frac{1}{\sin \theta} \partial_{\theta} h_{1}\right)\right), \\
\partial_{t} W+2 V W+U \partial_{\theta} W+U W \cot \theta-\omega W= \\
\frac{1}{R}\left(\frac{3}{\sin ^{2} \theta} h_{1} \partial_{\theta}\left(\sin \theta h_{2}\right)-\frac{2}{\sin \theta} \partial_{\theta} h_{1} h_{2}\right), \\
\partial_{\hat{t}}\left(\Pi R^{-\gamma}\right)+2 V \Pi R^{-\gamma}+U \partial_{\theta}\left(\Pi R^{-\gamma}\right)= \\
-2(\gamma-2) \omega \Pi R^{-\gamma}
\end{array}
$$

$6 \phi+\frac{1}{\sin \theta} \partial_{\theta}\left(\sin \theta \partial_{\theta} \phi\right)=-R$,

$\partial_{t} h_{1}+3 V(\theta) h_{1}(\theta)+U(\theta) \partial_{\theta} h_{1}(\theta)=2 \omega h_{1}(\theta)$,

$\partial_{t} h_{2}+3 V h_{2}-3 \partial_{\theta}\left(\frac{1}{\sin \theta} W\right) h_{1}+\partial_{\theta}\left(U h_{2}\right)=2 \omega h_{2}$.

The parameter $\lambda$, describing the radius rescaling, does not appear any longer in these equations because of the special dependence on $r$ stated by Eqs. (30) which indeed is scale invariant.

This set of equations (Eqs. (31)-(38)) is still quite complex and solving it completely is a formidable mathematical problem. We will thus look in the next sections for particular solutions of the system of Eqs. (31)-(38). More precisely, we will look for stationary solutions with respect to the $\widetilde{t}$ variable. Such solutions are not stationary with respect to the physical time $t$ and in spite of clear physical limitations, ought to describe an aspherical collapse or expansion with complex processes, like magnetic field or rotation included. 


\section{Exact solutions}

In this section, we derive exact solutions of Eqs. (31)-(38) stationary with respect to $\widetilde{t}$, (i.e. $\partial_{t}=0$ ) first in the hydrodynamical (i.e. unmagnetized) case: $h_{1}=h_{2}=0$ and then consider the MHD case. In Appendix A, the solutions are summarized and given explicitly in the physical space.

\subsection{Uniform density field: Gravitational potential and thermal pressure}

Equations (31), (32), (33), (35), and (36) cannot be easily integrated and in order to further reduce these equations, we look for solutions with uniform density:

$R(\theta)=1$.

\subsubsection{Gravitational potential}

One can easily obtain a solution of Eq. (36):

$\phi(\theta)=-\frac{1}{6}+\phi_{1}\left(3 \cos ^{2} \theta-1\right)$.

If $\phi_{1} \neq 0$, the gravitational potential is aspherical whereas the density field is constant. This result can be physically understood in the following way. The fields:

$$
\begin{aligned}
\widehat{\rho}_{\alpha}(\widehat{r}, \theta)= & \frac{1}{4 \pi G}(\alpha(\alpha+1) A \\
& \left.+(\alpha(\alpha+1)-6) B\left(3 \cos ^{2} \theta-1\right)\right) \widehat{r}^{\alpha-2},
\end{aligned}
$$

$\widehat{\Phi}_{\alpha}(\widehat{r}, \theta)=-\left(A+B\left(3 \cos ^{2} \theta-1\right)\right) \widehat{r}^{\alpha}$

are solutions of Eq. (21) ( $A$ and $B$ are two real numbers). The physical requirement $\widehat{\rho}_{\alpha}>0$ implies that:

$$
\begin{aligned}
& \alpha(\alpha+1) A+3 B(\alpha(\alpha+1)-6)>0 \\
& \alpha(\alpha+1) A-B(\alpha(\alpha+1)-6)>0 .
\end{aligned}
$$

In the limit $\alpha \rightarrow 2$, it is equivalent to $A>0$.

With these definitions, $R$ and $\phi$ are equal to respectively $\widehat{\rho}_{\alpha \rightarrow 2} / \rho_{0}$ and $\widehat{\Phi}_{\alpha \rightarrow 2} /\left(\Omega_{0}^{2} \widehat{r}^{2}\right)$. In the limit $\alpha \rightarrow 2$, the colatitude dependent part of $\widehat{\rho}_{\alpha}$ vanishes whereas this is not the case for $\widehat{\Phi}_{\alpha}$. This means, that in the limit $\alpha \rightarrow 2$, the colatitude dependent part of the density field stated by Eqs. (41) and (42) is dynamically negligible but not gravitationally negligible.

An explanation for the origin of the aspherical potential will be presented in Sect. 3.2.2.

If $\phi_{1}<-1 / 6$ then $\phi(0)<0$ and $\phi(\pi / 2)>0$. The gravitational force attracts towards the origin the gas at the pole and moves away from the origin the gas at the equator.

If $\phi_{1}>1 / 12$ then $\phi(0)>0$ and $\phi(\pi / 2)<0$. The gravitational force attracts towards the origin the gas at the equator and moves away from the origin the gas at the pole.
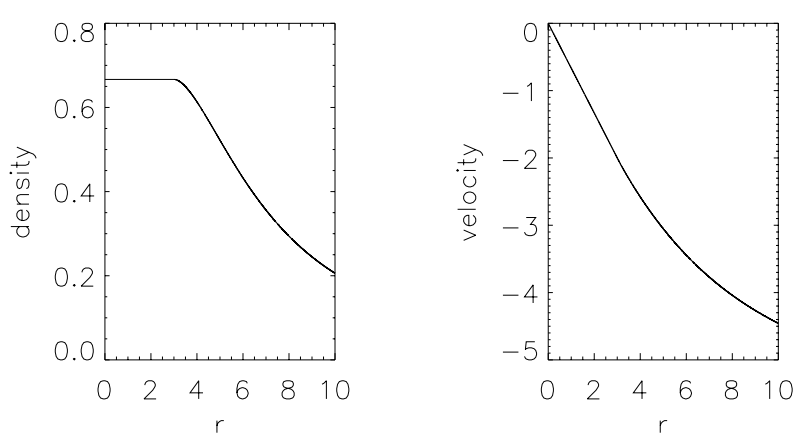

Fig. 1. Density and velocity fields corresponding to the LarsonPenston solution. The density is constant for $r<3$ and the velocity is homologous. A bifurcation occurs at the sonic point located at $r=3$.

\subsubsection{Thermal pressure}

The thermal pressure given by Eqs. (30) is proportional to $\Pi(\theta) \widehat{r}$, thus a negative value of $\Pi(\theta)$ could appear unphysical (negative thermal pressure). However, with the assumption stated by Eq. (39), it is easily seen that:

$P(t, r, \theta)=d(t)^{\gamma} P_{0}+\pi(t) \widehat{P}(\widehat{t}, \widehat{r}, \theta)$,

where $P_{0}$ is a real number, is also a solution of the problem $\left(d(t)^{\gamma} P_{0}(d(t) R)^{-\gamma}\right.$ is a constant). Thus choosing $P_{0}>0$, allows to have a thermal pressure positive until a finite value of $r$. It still becomes negative for an arbitrary high value of $r$, but with the assumption stated by Eqs. (30), as we already said, these solutions are valid only in a finite domain. The value of $P_{0}$ can thus be high enough for the thermal pressure to be positive in the whole domain of validity.

With the uniform density field assumption, $\widehat{P} \neq 0$ implies that the temperature is not a function of the density only as it is usually assumed. An anisotropic temperature can be due to anisotropic heating (Nelson \& Langer 1997 who consider an anisotropic UV heating), or to isotropic heating and anisotropic optical opacity resulting from the anisotropic shape of the cloud (see the discussion of Sect. 3.2.2 on the anisotropic sonic surface).

In Sect. 3 we will consider only the case where $\widehat{P}=0$. This implies that the thermal pressure does not have any dynamical effect since it is uniform. However, as for the Larson-Penston solution, it is expected that at the sonic point, i.e. when the velocity is equal to the sound speed, a bifurcation occurs (see Fig. 1). Thus, thermal pressure plays an important rôle since it induces the bifurcation and the solutions are not equivalent to the solutions describing the collapse of a cold cosmological cloud that has no thermal pressure (Lin et al. 1965).

In Sect. 4 , we will consider the case where $\widehat{P} \neq 0$.

\subsection{Hydrodynamical equations}

In this section, we consider the aspherical hydrodynamical case when the rotation is non zero and the gravitational potential depends on $\theta\left(\phi_{1} \neq 0\right)$. We look for solutions 

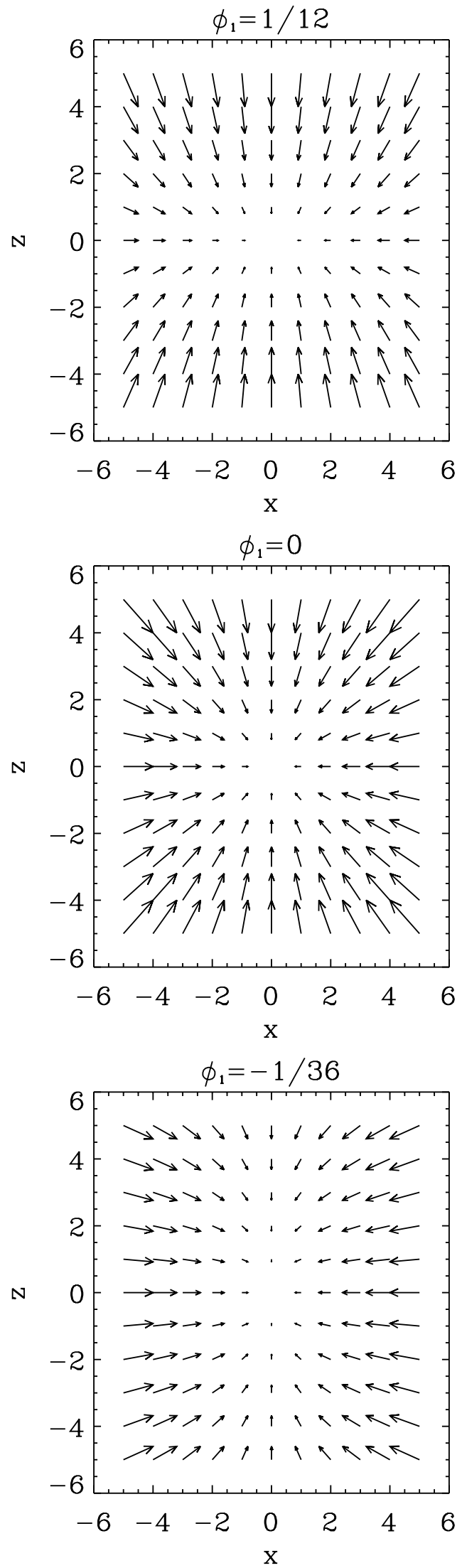

Fig. 2. Velocity field corresponding to collapse with anisotropic gravitational potential (solutions stated by Eqs. (48)) with $\phi_{1}=1 / 12$ (oblate sonic surface with aspect ratio of 2 ), 0 (spherical case), $-1 / 38$ (prolate sonic surface with aspect ratio of $5 / 2$ ). The field is axisymmetric with respect to the $z$-axis. with uniform density (Eq. (39)) and assume that:

$V(\theta)=V_{0}-U_{1} \cos ^{2} \theta, U(\theta)=U_{1} \cos \theta \sin \theta$,
$W(\theta)=W_{1} \sin \theta$.

Equations (31), (32), (33) and (34) lead to:

$$
\left\{\begin{array}{l}
U_{1}=3 V_{0}-2 \omega, \\
V_{0}^{2}-W_{1}^{2}-\omega V_{0}=-1 / 3-2 \phi_{1}, \\
-2 V_{0} U_{1}+U_{1}^{2}+W_{1}^{2}+\omega U_{1}=6 \phi_{1}, \\
W_{1}=0 \text { or } V_{0}=\omega / 2 .
\end{array}\right.
$$

The first equation derives from the mass conservation whereas the second and the third derive from the radial momentum conservation (the orthoradial momentum conservation leads also to the third equation). The term $W_{1}^{2}$ in the left-hand side of the second and the third equation is the centrifugal force. The fourth equation derives from the azimuthal momentum conservation.

With Eqs. (46), we obtain two solutions stated by Eqs. (48) and (51). The first one describes the collapse of an aspherical and non rotating cloud and the second one the collapse of a rotating and aspherical cloud.

\subsubsection{Spherical solution}

We first consider the spherical case with $U_{1}=0, W_{1}=0$ and $\phi_{1}=0$. We then have:

$V_{0}=2 / 3 \omega, \omega^{2}=3 / 2$,

which indeed is the solution found by Bouquet et al. (1985) and by Penston (1969a) and Larson (1969) in the isothermal case.

Bouquet et al. (1985) and Whitworth \& Summers (1985), in the case $\gamma=1$, show that at the sonic point, i.e when $\widehat{V}=\widehat{C}_{\mathrm{s}}\left(\widehat{C}_{\mathrm{s}}\right.$ being the sound speed $)$, the solution stated by Eq. (47), becomes unstable and a bifurcation occurs. After this point, the density decreases and one recovers asymptotically the Chandrasekhar solutions (Chandrasekhar 1967).

This is illustrated in Fig. 1 that displays the density field corresponding to the Larson-Penston solution. The bifurcation occurs at $r=3$.

\subsubsection{Aspherical gravitational potential}

If $W_{1}=0$ (no rotation) and $\phi_{1} \neq 0$ (anisotropic gravitational field), the solutions of Eqs. (46) are:

$$
\left\{\begin{array}{l}
\omega= \pm \sqrt{\frac{3}{2}} \frac{1+12 \phi_{1}}{\sqrt{1+24 \phi_{1}}}, U_{1}=-12 \sqrt{\frac{3}{2}} \frac{\phi_{1}}{\sqrt{1+24 \phi_{1}}} \frac{\omega}{|\omega|}, \\
V(\pi / 2)=V_{0}=\sqrt{\frac{2}{3}} \frac{1+6 \phi_{1}}{\sqrt{1+24 \phi_{1}}} \frac{\omega}{|\omega|}, \\
V(0)=\sqrt{\frac{2}{3}} \frac{1+24 \phi_{1}}{\sqrt{1+24 \phi_{1}}} \frac{\omega}{|\omega|} .
\end{array}\right.
$$

Due to an aspherical (see Eqs. (39), (40), (41), (42)) gravitational field the collapse or the expansion are aspherical. In the limit $\phi_{1} \rightarrow 0$, one recovers the solution stated by Eq. (47). A solution exist only if $\phi_{1}>-1 / 24$. 
It is likely (at least if $\phi_{1} \ll 1$ ) that as for the LarsonPenston solution a bifurcation will occur at the points of the sonic surface defined by: $\widehat{V}_{r}^{2}+\widehat{V}_{\theta}^{2}=\widehat{C}_{\mathrm{s}}^{2}$. With this definition, it is easily seen that the sonic surface is an ellipsoid defined by the relation:

$\frac{\Omega_{0}^{2} V_{0}^{2}}{\widehat{C}_{\mathrm{s}}^{2}}\left(x^{2}+y^{2}\right)+\frac{\Omega_{0}^{2}\left(V_{0}-U_{1}\right)^{2}}{\widehat{C}_{\mathrm{s}}^{2}} z^{2}=1$,

where $x=r \sin \theta \cos \phi, y=r \sin \theta \sin \phi$ and $z=r \cos \theta$. The length of its axes are: $a=\widehat{C}_{\mathrm{s}} /\left(\Omega_{0}\left|V_{0}\right|\right)$ and $b=$ $\widehat{C}_{\mathrm{s}} /\left(\Omega_{0}\left|V_{0}-U_{1}\right|\right)$, and the aspect ratio is given by:

$\frac{a}{b}=\frac{V_{0}-U_{1}}{V_{0}}=\frac{1+24 \phi_{1}}{1+6 \phi_{1}}$.

This fact, indeed leads to an explanation for the origin of the aspherical gravitational potential since the sonic surface is not spherical and since the gas density outside the sonic surface is not uniform.

If $\phi_{1} \rightarrow-1 / 24$ or $\phi_{1} \rightarrow \infty$, then $V(0) \rightarrow \infty$ which means that the sonic surface becomes smaller and tends to the origin.

We will not consider such bifurcation further even if this rather difficult (particularly in two dimensions) question requires an accurate and careful investigation at some stage.

If $\phi_{1}>0$ then $a / b>1$ and the cloud or at least the sonic surface is oblate. It is prolate in the other case. The velocity field corresponding to the three values $\phi_{1}=1 / 12$ (oblate cloud with aspect ratio equal to 2 ), $\phi_{1}=0$ (spherical solution), $\phi_{1}=-1 / 36$ (prolate cloud with aspect ratio equal to 5/2) are displayed in Fig. 2.

\subsubsection{Rotation}

We now consider a non vanishing azimuthal velocity.

The parameters of the solution are:

$\left\{\begin{array}{l}\omega= \pm \sqrt{4 / 3+8 \phi_{1}-4 W_{1}^{2}}, \\ \phi_{1}=1 / 12 ; U_{1}=-\omega / 2, \\ V(\pi / 2)=V_{0}=\omega / 2, V(0)=\omega .\end{array}\right.$

The gravitational potential is strongly constrainted: $\phi_{1}=$ $1 / 12$, and $\phi(0)=0$. The sonic surface is oblate with an aspect ratio of 2 , the minor axis being parallel to the rotation axis. The frequency, $\omega$, decreases with increasing rotation velocity and the solution becomes stationary for $W_{1}=1 / \sqrt{2}$, meaning that for this value the cloud is rotationaly supported.

\subsection{Magnetohydrodynamics}

In this part, we consider the gravo-magnetic condensation and derive exact solutions.

If $\partial_{t}=0$, Eqs. (31) and (37) lead to:

$K R(\theta) U(\theta) \sin \theta=h_{1}(\theta)$,

where $K$ is a real number.

We consider first a problem without rotation and without magnetic toroidal field.
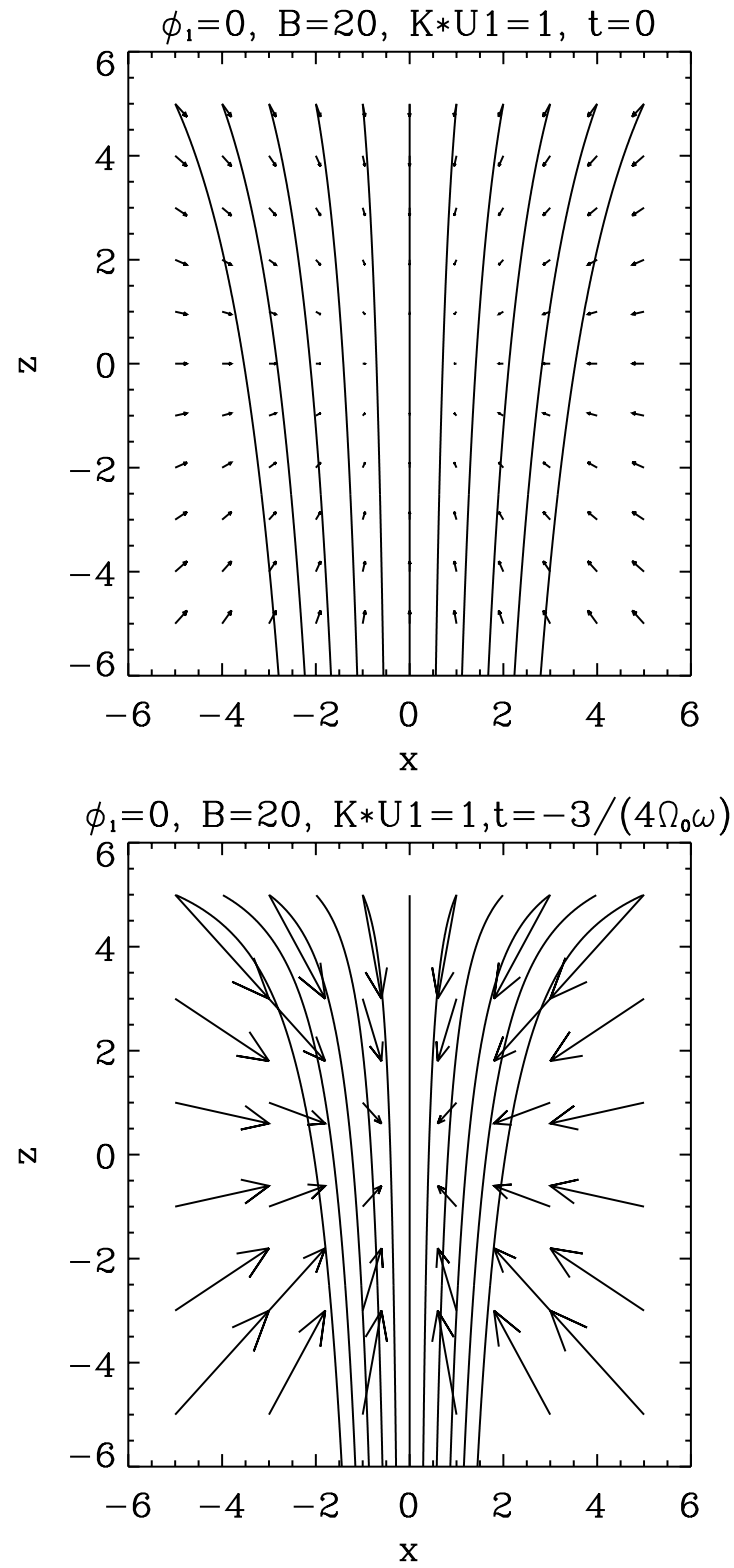

Fig. 3. Velocity field and magnetic field lines corresponding to Eqs. (47) and to Eqs. (58)-(59) (with $B=20$ and $K U_{1}=1$ ). The two time steps, $t=0$ and $t=3 /\left(4 \Omega_{0}|\omega|\right)$, are displayed. The evolution of the velocity field is self-similar and it is multiplied by 4 between the 2 steps. The evolution of the magnetic field is not self-similar because the uniform component and the component stated by Eqs. (54) have different temporal evolution.

\subsubsection{Magnetic field without rotation}

We consider a magnetized, non rotating cloud and we look for solutions stated by Eqs. (45). With Eq. (52), we have:

$h_{1}(\theta)=K U_{1} \cos \theta \sin ^{2} \theta$.

The corresponding magnetic components are proportional to:

$B_{r} \propto-\left(3 \cos ^{2} \theta-1\right) r$,

$B_{\theta} \propto 3 \cos \theta \sin \theta r$. 
We have:

$\frac{6}{\sin \theta} h_{1}+\partial_{\theta}\left(\frac{1}{\sin \theta} \partial_{\theta} h_{1}\right)=0$

which means that $\operatorname{rot} \boldsymbol{B}=0$. Thus, for the field given by Eq. (53) the Lorentz force vanishes (see Eqs. (32)-(33)).

Before continuing, we demonstrate that adding a spatialy uniform magnetic field is possible. Let us consider the uniform magnetic field:

$$
\begin{aligned}
& B_{r}(t, r, \theta)=b(t) B \cos \theta, \\
& B_{\theta}(t, r, \theta)=-b(t) B \sin \theta
\end{aligned}
$$

where $B$ is a magnetic intensity. Here again the Lorentz force vanishes. With Eqs. (6) and for the velocity field stated by Eq. (45), one finds that:

$b(t)=\left(1+\Omega_{0} \omega t\right)^{-2 V_{0} / \omega}$.

Consequently, Eqs. (1)-(6) admit the solutions given by Eqs. (48) (and by Eqs. (12), (25), (30), (45)) with the magnetic field (since the Lorentz force associated to the fields stated by Eqs. (54) and (56) vanishes):

$$
\begin{aligned}
& B_{r}(t, r, \theta)=B\left(1+\Omega_{0} \omega t\right)^{-2 V_{0} / \omega} \cos \theta \\
& +\sqrt{\mu_{0} \rho_{0}} \times \Omega_{0}\left(1+\Omega_{0} \omega t\right)^{-2} K\left(-3 U_{1} \cos ^{2} \theta+U_{1}\right) r, \\
& B_{\theta}(t, r, \theta)=-B\left(1+\Omega_{0} \omega t\right)^{-2 V_{0} / \omega} \sin \theta \\
& +\sqrt{\mu_{0} \rho_{0}} \times \Omega_{0}\left(1+\Omega_{0} \omega t\right)^{-2} K\left(3 U_{1} \cos \theta \sin \theta\right) r,
\end{aligned}
$$

for any value of $B$ and $K$. In the limit $U_{1} \rightarrow 0$ (reached for $\phi_{1} \rightarrow 0$ in Eqs. (48) and $\gamma \rightarrow 4 / 3$ in Eqs. (B.1) and (B.2)) and $K \rightarrow \infty$, one finds that they also admit the spherical solutions (Eqs. (47)).

Figure 3 displays the velocity field and magnetic field lines stated by Eqs. (47) with the magnetic fields stated by Eqs. (58)-(59) $\left(\phi_{1}=0, B=20, K U_{1}=1\right)$ at two times steps $\left(t=0\right.$ and $\left.t=-3 /\left(4 \Omega_{0} \omega\right)\right)$. The behaviour of the magnetic field is not self-similar because the two components of Eqs. (58)-(59) are differentially rescaled.

\subsubsection{Rotation and magnetic field}

In this section we consider a rotating and magnetized cloud. We look for solutions stated by Eqs. (45), (53) and:

$h_{2}(\theta)=Q \sin \theta$.

The corresponding azimuthal magnetic component is proportional to:

$B_{\phi} \propto \sin \theta r$.

Equations (31), (32), (33) and (34) lead to:

$$
\left\{\begin{array}{l}
U_{1}=3 V_{0}-2 \omega, \\
V_{0}^{2}-W_{1}^{2}-\omega V_{0}=-1 / 3-2 \phi_{1}-2 Q^{2}, \\
-2 V_{0} U_{1}+U_{1}^{2}+W_{1}^{2}+\omega U_{1}=6 \phi_{1}+2 Q^{2}, \\
V_{0} W_{1}-\omega W_{1} / 2=K Q U_{1},
\end{array}\right.
$$

whereas Eq. (38) gives the same constraint as Eq. (31).
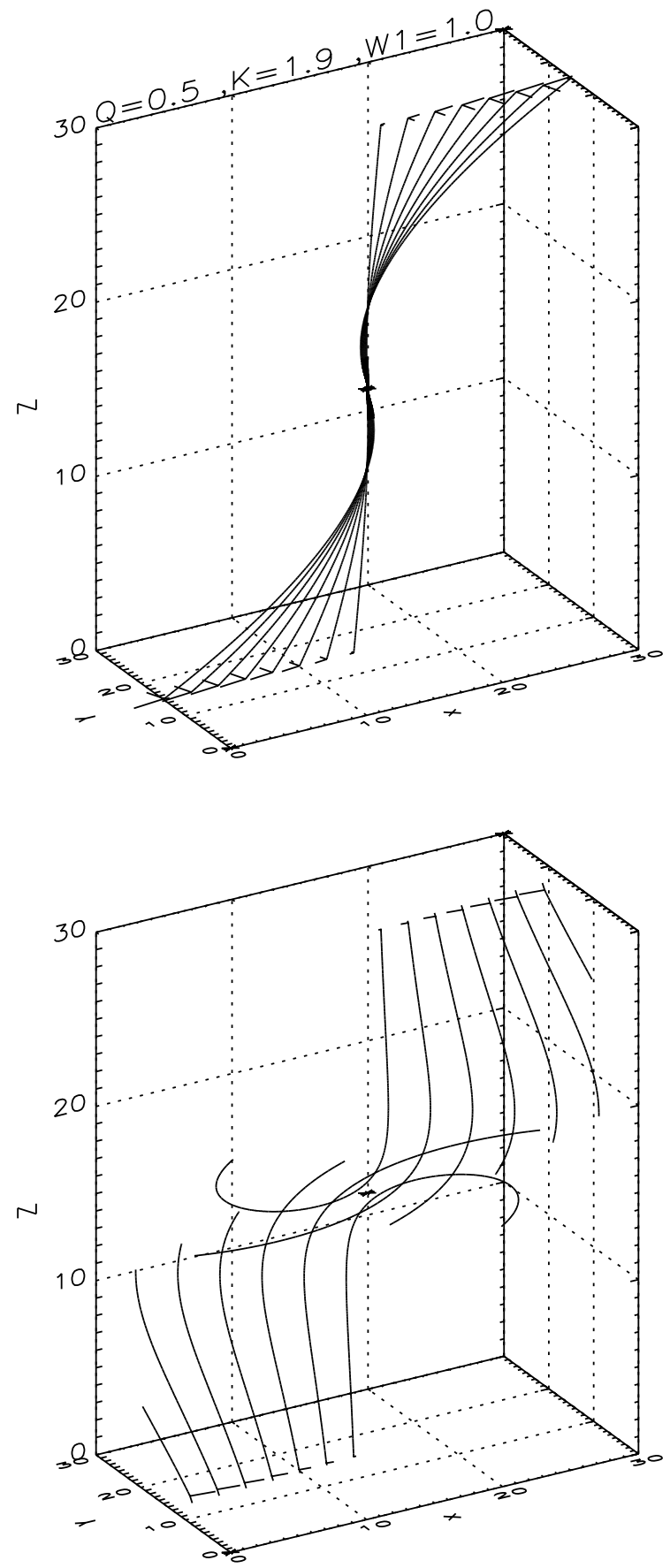

Fig. 4. Stream lines (upper panel) and magnetic field lines (lower panel) of the fields corresponding to Eqs. (63) $(B=0)$ with parameters corresponding to the weak rotation regime (first case). The converging center $(r=0)$ is located in the box centre $(x=15, y=15, z=15)$. The gas is accreted at the pole and at the equator.

The fourth equation derives from the azimuthal momentum conservation, its right-hand side is the magnetic tension which is proportional to the product of the poloidal and toroidal field. The term $Q^{2}$ in the right-hand side of the second and the third equations is the Lorentz force due to the toroidal field. It tends to compress the gas because the magnetic toroidal intensity increases with the 

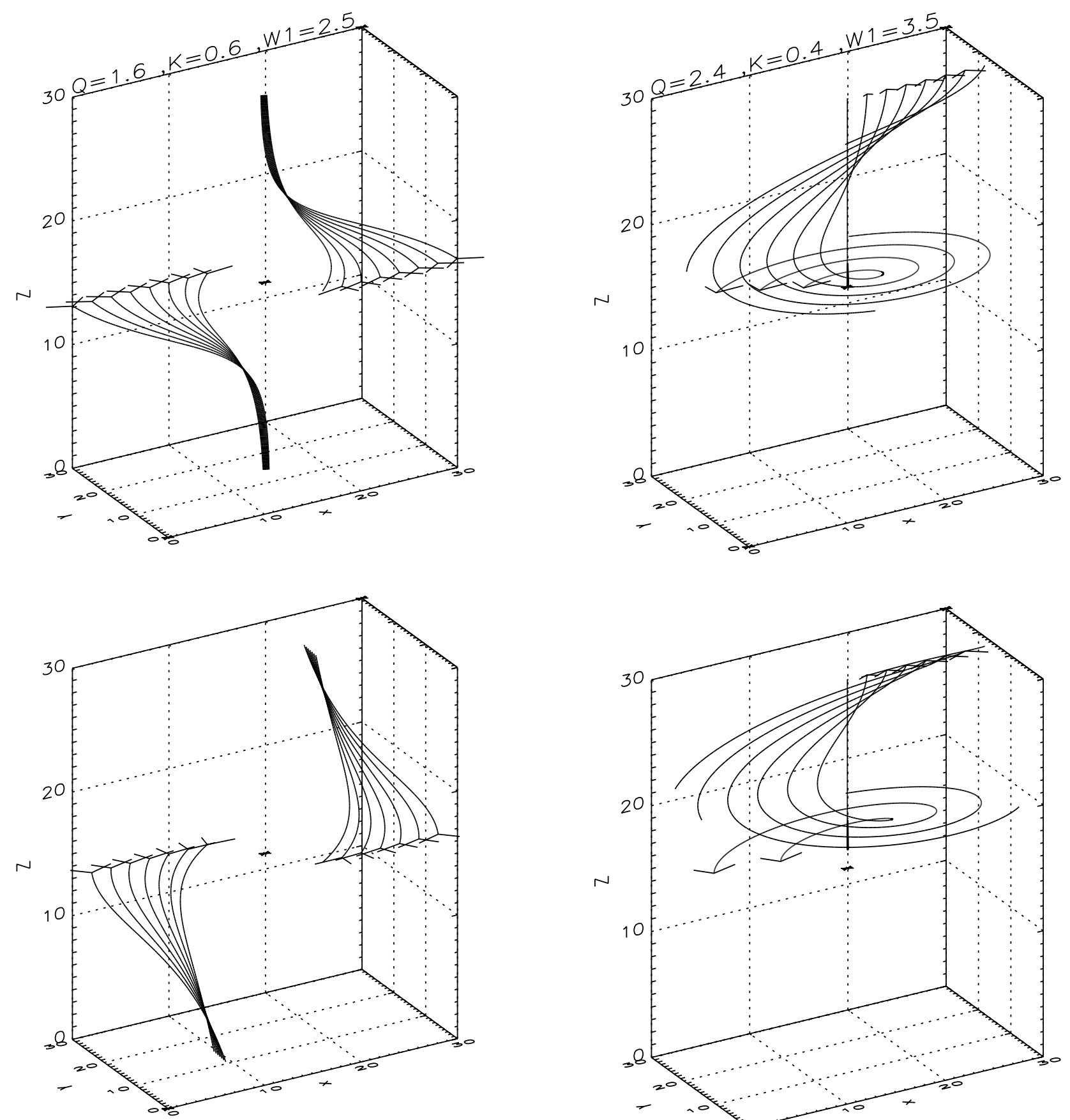

Fig. 5. Same as Fig. 4 for parameters corresponding to the second case (strong rotation): $2 Q K<W_{1}<3 Q K$. The gas is accreted at the equator and ejected at the pole.

distance to the $z$-axis, $\varpi$. There is no magnetic term due to the poloidal field only (proportional to $K^{2}$ ) because its contribution vanishes (see Eq. (55)).

As in the non-rotating case, one can easily check that adding a uniform magnetic field (Eq. (56)) is still possible (the terms $(-\sin \theta) \partial_{\theta}\left(\sin \theta B_{\phi}\right)+\cos \theta \sin \theta \partial_{r}\left(r B_{\phi}\right)$ in Eq. (19) and $\partial_{r}\left(r V_{\phi} \cos \theta\right)+\partial_{\theta}\left(V_{\phi}(-\sin \theta)\right)$ in Eq. (6) cancel). The physical meaning is that a solid body rotation does not stretch a uniform field parallel to the rotation axis and an helicoidal field (uniform poloidal field along

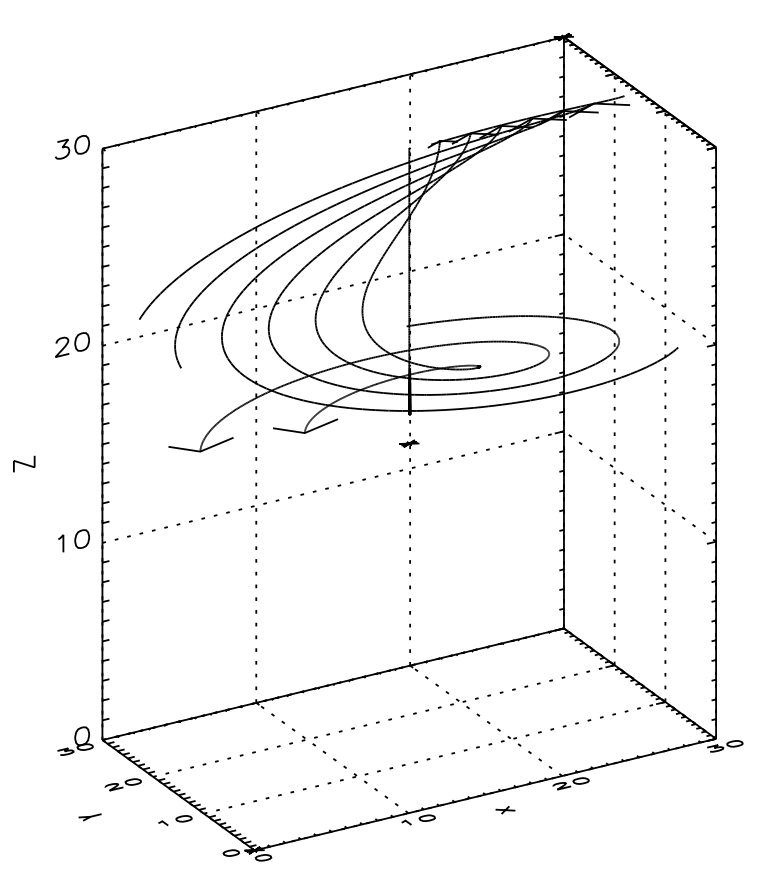

Fig. 6. Same as Fig. 4 for parameters corresponding to the third case (strong rotation): $3 Q K<W_{1}<4 Q K$. The gas is accreted at the pole and ejected at the equator.

the $z$-axis and toroidal field proportional to $\varpi$ ) does not induce any azimuthal tension.

Consequently, one obtains the poloidal magnetic field given by Eqs. (58)-(59) whereas the toroidal field is given by Eq. (61).

The solution of Eq. (62) is given by:

$$
\left\{\begin{array}{l}
\omega= \pm \sqrt{\frac{\left(2+8 Q^{2}-4 W_{1}^{2}\right)\left(W_{1}-3 Q K\right)^{2}}{\left(W_{1}-2 Q K\right)\left(W_{1}-6 Q K\right)}} \\
\phi_{1}=1 / 12+\omega^{2} \frac{Q K W_{1}-2(Q K)^{2}}{4\left(W_{1}-3 Q K\right)^{2}}, U_{1}=-\frac{W_{1}}{W_{1}-3 Q K} \omega / 2, \\
V(\pi / 2)=V_{0}=\frac{W_{1} / 2-2 Q K}{W_{1}-3 Q K} \omega, V(0)=\frac{W_{1}-2 Q K}{W_{1}-3 Q K} \omega
\end{array}\right.
$$




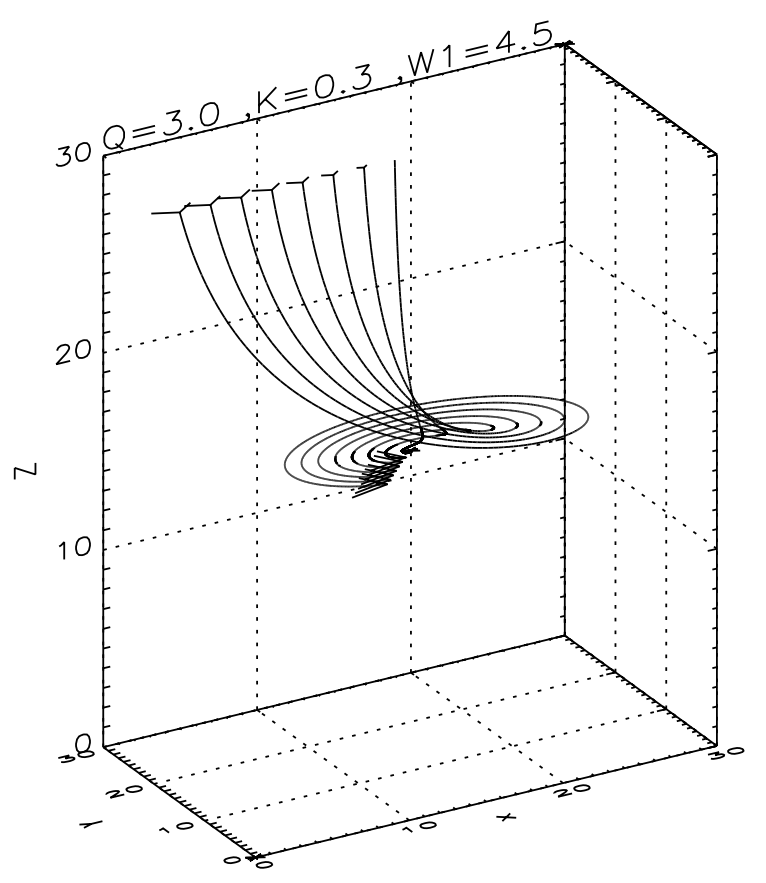

The gravitational potential is spherical $\left(\phi_{1}=0\right)$ if $W_{1}+12 Q K\left(2 Q^{2}-W_{1}^{2}\right)=0$. We have $V(0) \omega>0$ and $V(\pi / 2) \omega>0$ (inflow).

If $Q K U_{1} \leq 0$, the azimuthal magnetic tension tends to decrease the azimuthal velocity (see Eqs. (62)). This is the magnetic braking effect. If $Q K U_{1} \geq 0$, the magnetic tension increases the rotation velocity. This forcing could be related to the Torsional Alfvén waves inducing collapse studied by Habe et al. (1991).

This regime is illustrated in Fig. 4 that displays the stream lines and magnetic field lines for $W_{1}=1, Q=0.52$ and $K=1.9$.

$-W_{1}>\sqrt{1 / 2+2 Q^{2}}$ (fast rotation) and $2 Q K<W_{1}<$ $6 Q K$. The centrifugal force dominates gravity (more precisely the spherical part of the gravitational force) and magnetic toroidal pinching. $\phi_{1}$ is greater than $1 / 12$ and thus gravity tends to move away from the origin the gas at the equator (see Sect. 3.1.1).

Assuming that, $2 Q K>\sqrt{1 / 2+2 Q^{2}}$, three cases have to be distinguished:

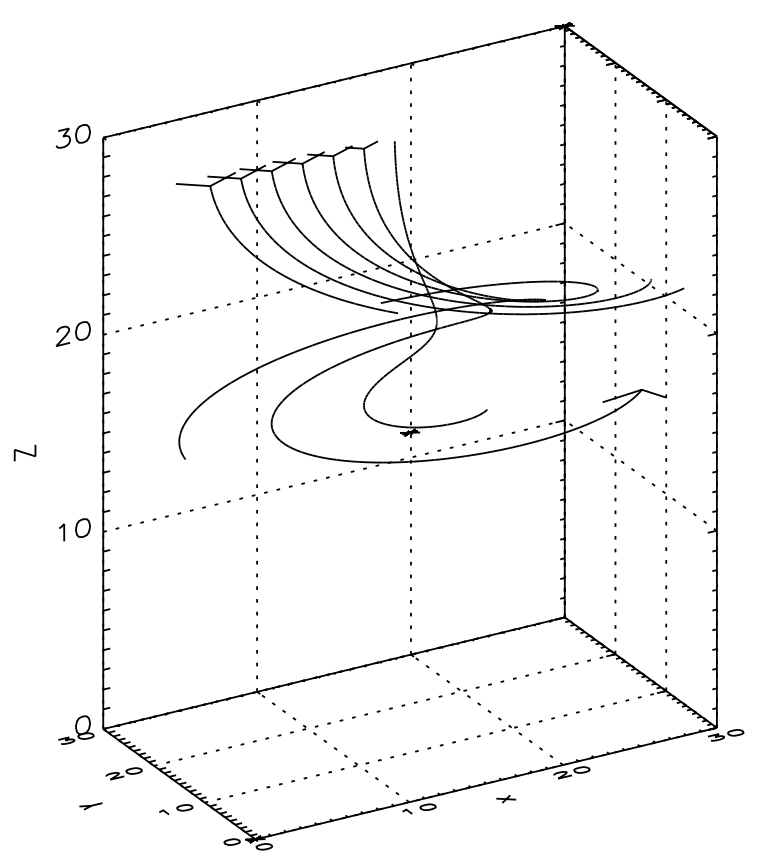

- $2 Q K<W_{1}<3 Q K$ then $V(0) \omega<0$ and $V(\pi / 2) \omega>0$, $\omega<0$ (collapse) implies that the gas is ejected at the pole (outflow) and accreted at the equator (inflow). Figure 5 displays the stream lines and magnetic field lines for $W_{1}=2.5, Q=1.6$ and $K=0.6$.

As for the weak rotation case with $Q K U_{1} \leq 0$, the azimuthal magnetic tension tends to brake the clouds. Since $\phi_{1}>1 / 12$, the gravitational potential becomes positive at the pole and gravity is responsible of the gas ejection along the pole (gravity is the only force along the $z$-axis). It is not clear if this is the same physical mechanism that is responsible for outflows found in numerical simulations of collapse-driven outflow (e.g. Tomisaka 1998, 2001).

- $3 Q K<W_{1}<4 Q K$ then $V(0) \omega>0$ and $V(\pi / 2) \omega<0$, $\omega<0$ (collapse) implies that the gas is accreted (inflow) at the pole and ejected (outflow) at the equator. Figure 6 displays the stream lines and magnetic field lines for $W_{1}=3.5, Q=2.4$ and $K=0.4$,

Since $U_{1} Q K \geq 0$, the magnetic tension tends to increase the cloud rotation velocity.

Fig. 7. Same as Fig. 4 for parameters corresponding to the fourth case (strong rotation): $4 Q K<W_{1}<6 Q K$. The gas is accreted at the equator and at the pole.

$W_{1}, Q$ and $K$ are free parameters. The transformation $W_{1} \rightarrow-W_{1}, Q K \rightarrow-Q K$ leaves the solution invariant and we can assume without restriction that $W_{1}>0$.

Two cases arise (conditions for $\omega^{2}$ to be positive):

$-W_{1}<\sqrt{1 / 2+2 Q^{2}}$ (weak rotation) and $W_{1}<2 Q K$ or $W_{1}>6 Q K$. The centrifugal force is smaller than the contribution of the gravity and the toroidal magnetic forces.

- $4 Q K<W_{1}<6 Q K$ then $V(0) \omega>0$ and $V(\pi / 2) \omega>0$, $\omega<0$ (collapse) implies that the gas is ejected at the pole and at the equator (usual behaviour). Figure 7 displays the stream lines and magnetic field lines for $W_{1}=4.5, Q=3$ and $K=0.3$.

As for the previous case, the magnetic tension tends to increase the cloud rotation velocity.

In the limit $W_{1} \rightarrow 3 Q K$, one finds the surprising result that $\omega \rightarrow 0$ (stationary solution) but, if $18 Q^{2} K^{2}-4 Q^{2}-$ $1>0$ :

$|V(\pi / 2)| \rightarrow 1 / \sqrt{6} \sqrt{18 Q^{2} K^{2}-4 Q^{2}-1} \neq 0$,
$|V(0)| \rightarrow \sqrt{2 / 3} \sqrt{18 Q^{2} K^{2}-4 Q^{2}-1} \neq 0$,
$\left|U_{1}\right| \rightarrow \sqrt{3 / 2} \sqrt{18 Q^{2} K^{2}-4 Q^{2}-1} \neq 0$ 
with $V(0) V(\pi / 2)<0$ and where the sign of $V(0)$ can be positive or negative. The physical meaning is that the outflow rate is equal to the inflow rate. Indeed the accretion rate, $J$, is proportional to:

$J(r)=2 \int_{0}^{\pi / 2} 2 \pi r^{2} \sin \theta \rho V_{r} \mathrm{~d} \theta \propto V_{0}-U_{1} / 3$

and consequently vanishes (see Eqs. (64)).

Let us summarize the results.

- We find four different regimes, one corresponds to a low rotation and three to a strong rotation (greater than gravity and magnetic toroidal forces). For two sets of parameters, one finds outflows at the pole or at the equator;

- In the weak rotation regime with $Q K U_{1} \leq 0$ and in the first case of the strong rotation regime, the magnetic tension brakes the cloud. In the other cases, it tends to accelerate the cloud rotation.

Although the magnetic field stated by Eqs. (58), (59) and (61) is not force-free, since the azimuthal tension and the magnetic toroidal pinching do not vanish, the poloidal magnetic pressure is equal to zero and does not support the cloud as it is the case in the numerical simulations of gravo-magnetic collapse which generally assume uniform initial mass-to-magnetic flux ratio. This fact clearly limits the applicability of the solutions to collapse for which the magnetic poloidal support is not dominant (weak magnetic field or field dominated by the toroidal component).

\section{Aspherical temperature}

In this section we briefly consider the case of an aspherical thermal pressure. We also extend the angular dependence of the various fields and find solutions that present velocity fields not symmetrical with respect to the equatorial plan.

With the uniform density assumption, as we already said in Sect. 3.1.2, this means that the temperature is not a function of the density only as it is usually assumed. Although less astrophysically relevant than the uniform temperature cases studied in the previous section, such temperature gradients are expected when radiative processes are considered.

\subsection{Results}

We look for solutions given by:

$\left\{\begin{array}{l}V(\theta)=V_{0}-U_{1} \cos ^{2} \theta-U_{2} \cos \theta \sin \theta \\ U(\theta)=U_{1} \cos \theta \sin \theta+U_{2} \sin ^{2} \theta \\ \Pi(\theta)=\Pi_{0}+\Pi_{1} \cos ^{2} \theta+\Pi_{2} \cos \theta \sin \theta \\ h_{1}(\theta)=K\left(U_{1} \cos \theta \sin ^{2} \theta+U_{2} \sin ^{3} \theta\right) \\ W(\theta)=W_{1} \sin \theta, h_{2}(\theta)=Q \sin \theta\end{array}\right.$

We then have:

$B_{r} \propto\left(-3 U_{1} \cos ^{2} \theta+U_{1}-3 U_{2} \cos \theta \sin \theta\right) r$,

$B_{\theta} \propto\left(3 U_{1} \cos \theta \sin \theta+3 U_{2} \sin ^{2} \theta\right) r$,
As physically expected, the azimuthal and orthoradial components of the magnetic and velocity fields vanish at the pole.

Equations (31)-(34) give:

$$
\left\{\begin{array}{l}
U_{1}=3 V_{0}-2 \omega, \\
V_{0}^{2}-\omega V_{0}-W_{1}^{2}= \\
\quad-2 \Pi_{0}-1 / 3-2 \phi_{1}-9 K^{2} U_{2}^{2}-2 Q^{2} \\
-2 V_{0} U_{1}+U_{1}^{2}+\omega U_{1}+W_{1}^{2}= \\
\quad-2 \Pi_{1}+6 \phi_{1}+9 K^{2} U_{2}^{2}+2 Q^{2}, \\
-2 V_{0} U_{2}+U_{2} U_{1}+\omega U_{2}=-2 \Pi_{2}-9 K^{2} U_{1} U_{2}, \\
\Pi_{2}+6 K^{2} U_{1} U_{2}=0, \\
V_{0} W_{1}-\omega W_{1} / 2=K Q U_{1} .
\end{array}\right.
$$

The second, third and fourth equations derive from the radial momentum conservation and the third, the fourth and the fifth from the orthoradial momentum conservation. The fifth equation shows that there is a non vanishing orthoradial component of the Lorentz force at the pole that must be counterbalanced by the thermal pressure.

Equation (35) leads to:

$$
\left\{\begin{aligned}
\left(-2 U_{1}\right) \Pi_{0}+ & \left((2 \gamma-4) \omega+2 V_{0}-2 U_{1}\right) \Pi_{1} \\
\left(-2 U_{2}\right) \Pi_{0}+ & \left(-2 U_{2}\right) \Pi_{1}+\quad \Pi_{2}=0 \\
& \left((2 \gamma-4) \omega+2 V_{0}-U_{1}\right) \Pi_{2}=0 \\
((2 \gamma-4) \omega+ & \left.2 V_{0}\right) \Pi_{0}+\left(-U_{2}\right) \Pi_{2}=0
\end{aligned}\right.
$$

One obtains the four solutions:

$$
\left\{\begin{array}{l}
\Pi_{0}=-\Pi_{1},(\gamma-2) \omega+V_{0}=0, \Pi_{2}=0, U_{2}=0 \\
\Pi_{0}=0,(\gamma-2) \omega+V_{0}-U_{1}=0, \Pi_{2}=0, U_{2}=0 \\
U_{2} \Pi_{2}=2 U_{1} \Pi_{0},\left(U_{1}^{2}-U_{2}^{2}\right) \Pi_{2}=2 U_{1} U_{2} \Pi_{1}, \\
\quad(\gamma-2) \omega+V_{0}=U_{1}, \\
\Pi_{0}+\Pi_{1}=0, U_{2} \Pi_{2}=U_{1} \Pi_{0}, 2(\gamma-2) \omega+2 V_{0}=U_{1} .
\end{array}\right.
$$

The first and the second solutions are symmetrical with respect to the equatorial plane whereas the third and the fourth are not.

\subsection{Non-symmetrical solutions with respect to the equatorial plan}

In this section we give and discuss the two solutions of Eqs. (68) and Eqs. (70) with $U_{2} \neq 0$ and $\Pi_{2} \neq 0$ as they present interesting physical features. The solutions symmetrical with respect to the equatorial plan are given for completeness in Appendix B.

These two solutions are given by:

$$
\begin{aligned}
& \left\{\begin{array}{l}
\Pi_{0}+\Pi_{1}=-3 K^{2} U_{1}^{2}, U_{2} \Pi_{2}=2 U_{1} \Pi_{0}, \Pi_{0}=\frac{2-\gamma}{3 \gamma-4} U_{2}^{2} \\
\omega= \pm \sqrt{\frac{1-2 \Pi_{0}+4 Q^{2}-2 W_{1}^{2}}{2-\gamma}} \\
\phi_{1}=1 / 12-\omega^{2}(2-\gamma)^{2} / 8 \\
K^{2}=\frac{1}{3} \frac{2-\gamma}{4-3 \gamma}, W_{1}=K Q \frac{3 \gamma-4}{\gamma-1}, U_{1}=(3 / 2 \gamma-2) \omega \\
V(\pi / 2)=V_{0}=\gamma \omega / 2, V(0)=(2-\gamma) \omega
\end{array}\right. \\
& \left\{\begin{array}{l}
\Pi_{0}+\Pi_{1}=0, U_{2} \Pi_{2}=U_{1} \Pi_{0}, \Pi_{0}=-6 K^{2} U_{2}^{2} \\
\omega= \pm \sqrt{\frac{-1-\Pi_{0}-4 Q^{2}+2 W_{1}^{2}}{2(2 \gamma-3)(6 \gamma-7)}} \\
\phi_{1}=1 / 12+\omega^{2}(2 \gamma-3)(4 \gamma-5) / 2 \\
K^{2}=\frac{1}{6} \frac{3-2 \gamma}{4-3 \gamma}, W_{1}=4 K Q \frac{4-3 \gamma}{5-4 \gamma}, U_{1}=2(3 \gamma-4) \omega \\
V(\pi / 2)=V_{0}=2(\gamma-1) \omega, V(0)=2(3-2 \gamma) \omega
\end{array}\right.
\end{aligned}
$$


For the first solution (Eqs. (71)), $K^{2}>0 \Rightarrow \gamma<4 / 3$, $\phi_{1}<1 / 12$ and $W_{1}^{2}<1 / 2-\Pi_{0}+2 Q^{2}$.

The rotation parameter, $W_{1}$, is a function of the toroidal magnetic intensity, $Q$, and the polytropic index $\gamma$. The rotation tends to prevent the condensation whereas the magnetic field, tends to compress the gas (see the terms $2 W_{1}^{2}$ and $-4 Q^{2}$ in the expression of $\omega$ ) and it is worthwhile to know the global trend of both effects. We have:

$-4 Q^{2}+2 W_{1}^{2}=-2 / 3 Q^{2} \frac{3 \gamma^{2}-2 \gamma-2}{(\gamma-1)^{2}}$.

If $\gamma<(1+\sqrt{7}) / 3$ one has: $-4 Q^{2}+2 W_{1}^{2}>0$ which means that for the solution stated by Eqs. (71), the global effect of the rotation and the magnetic compression is to prevent the collapse. If $\gamma>(1+\sqrt{7}) / 3$ one has: $-4 Q^{2}+2 W_{1}^{2}<0$.

For the second solution (Eqs. (72)), one has: $K^{2}>0 \Rightarrow$ $\gamma<4 / 3$ or $\gamma>3 / 2$.

We have:

$-4 Q^{2}+2 W_{1}^{2}=-4 / 3 Q^{2} \frac{24 \gamma^{2}-52 \gamma+27}{(5-4 \gamma)^{2}}$

and

$-4 Q^{2}+2 W_{1}^{2}>0 \Leftrightarrow$

$$
(13-\sqrt{7}) / 12<\gamma<(13+\sqrt{7}) / 12 .
$$

Thus if $(13-\sqrt{7}) / 12<\gamma<(13+\sqrt{7}) / 12$ then $-4 Q^{2}+$ $2 W_{1}^{2}-\Pi_{0}-1$ can be positive even with $\Pi_{0}>0$ if $Q$ is large enough, whereas in the opposite case, only negative values of $\Pi_{0}$ (the thermal pressure tends to compress the gas) are possible in order for $-4 Q^{2}+2 W_{1}^{2}-\Pi_{0}-1$ to be positive.

Two cases arise:

$--4 Q^{2}+2 W_{1}^{2}-\Pi_{0}<1$ (weak magnetic intensity/rotation and/or weak aspherical thermal pressure), implies $7 / 6<\gamma<4 / 3$;

One has $V(0) \omega>0$ and $V(\pi / 2) \omega>0$;

$--4 Q^{2}+2 W_{1}^{2}-\Pi_{0}>1$ (strong magnetic intensity/rotation and/or strong aspherical thermal pressure), implies $\gamma<7 / 6$ or $\gamma>3 / 2$;

- If $\gamma<1$, one has $V(\pi / 2) \omega<0$ and $V(0) \omega>0, \omega<0$ (collapse) implies that the gas is accreted (inflow) at the pole and ejected (outflow) at the equator;

- If $1<\gamma<7 / 6, \omega<0$ (collapse) implies that the gas is accreted at the pole and at the equator (inflow);

- If $\gamma>3 / 2, V(\pi / 2) \omega>0$ and $V(0) \omega<0, \omega<0$ (collapse) implies that the gas is ejected (outflow) at the pole and accreted (inflow) at the equator.

As for Sect. 3.3.2, one finds that the outflow solutions have $\phi_{1}>1 / 12$ (positive gravitational potential at the pole).

\section{Conclusion}

In this paper, we apply the transformation proposed by Munier \& Feix (1983) to the axisymmetric magnetohydrodynamical equations of a self-gravitating polytropic gas.
We reduce these equations to a system of 8 ordinary differential equations of the variable $\theta$ (with Eq. (53) it is reduced to 7 equations only). We then derive exact and explicit self-similar solutions of these ordinary differential equations capable to describe the gravitational collapse of a uniform density cloud with aspherical pressure, aspherical gravitational potential, magnetic field and rotation. These solutions generalise the Larson-Penston solutions in the case where these processes are considered and thus describe the subsonic core of a collapsing cloud (Whitworth \& Summers 1985).

For the solutions of the collapse with an aspherical gravitational potential, we show that the sonic surface is an ellipsoid that can be oblate or prolate. Assuming that a bifurcation occurs at this surface (as it is the case for the spherical solution), this suggests that these solutions describe the internal part of elliptical clouds.

We find that the solutions given by Eqs. (47), (48), (B.1) and (B.2) describing the collapse of a non rotating cloud, are compatible with a magnetic field given by Eqs. (58) and (59) of any intensity since the magnetic configuration is such that the Lorentz force vanishes.

The solutions describing the collapse of magnetized and rotating clouds with uniform thermal pressure (Eqs. (63)) present four regimes, one corrresponding to slow rotation and three to rapid rotation. The solutions corresponding to these three regimes (rapid rotation) present (depending on the rotation and on the magnetic intensity) outflow at the pole, outflow at the equator or inflow only. There is a value of the rotation (Eqs. (64)) for which the outflow rate is equal to the inflow rate and the solution is stationary $(\omega=0)$.

Four regimes, depending on $\gamma$, are also found for one of the (non symmetrical with respect to the equatorial plane) solutions describing the collapse of a magnetized and rotating cloud with aspherical pressure (Eq. (72)). One regime $(\gamma<1)$ presents outflow at the equator, one regime $(\gamma>3 / 2)$ outflow at the pole.

The two main restrictions of the present work are, first the Lorentz force due to the poloidal magnetic field vanishes (if $U_{2}=0$ ) and thus, the magnetic poloidal force is not a support against the gravitational collapse (if $U_{2} \neq 0$, it compresses the gas), second the solutions diverge when $r$ goes to infinity and are valid in a finite domain only. The study of the bifurcation that could occur at the sonic (or magneto-sonic) point has not been considered here but must be carefully addressed. Indeed, a numerical solution of the two dimensional stationary system of Eqs. (16)(23) (see e.g. Arthur \& Falle 1991 who carry out a similar study for a bidimensional self-similar supernovae explosion) must be addressed at some stage. It should make possible to avoid the vanishing poloidal force and should give the solution on the whole domain. Such a study should give a sensible hint of the gravo-magnetic condensation.

Nevertheless, these solutions that generalise the Larson-Penston solution have an explicit dependence on the radius $r$ and the colatitude $\theta$ and present a wide class of physical behaviours. They offer an opportunity for the 
testing of numerical codes and a starting point for future analytical studies.

Acknowledgements. I thank Serge Bouquet for helpful and stimulating discussions. I thank referees Frank Shu and Shantanu Basu for their help in improving the manuscript. I also acknowledge a critical and helpful reading of the manuscript by Serge Bouquet, Anthony Whitworth, Robin Williams and Michel Pérault.

\section{Appendix A: Exact and explicit solutions of the gravo-magnetic condensation}

In this appendix we summarize the solutions found in this paper and give them explicitly. The values of the parameters $\gamma, \omega, \Pi_{0}, \Pi_{1}, \Pi_{2}, U_{1}, U_{2}, V_{0}, \phi_{1}, K$ and $Q$ being given by Eqs. (47), (48), (B.1), (B.2), (51), (B.3), (B.4), (63), (B.5), (B.6), (71) or $(72), \rho_{0}$ and $P_{0}$ being free parameters and $\Omega_{0}=\sqrt{4 \pi G \rho_{0}}$, Eqs. (1)-(6) admit the solutions given by:

$$
\begin{aligned}
\rho(t, r, \theta)= & \frac{\rho_{0}}{\left(1+\Omega_{0} \omega t\right)^{2}}, \\
\Phi(t, r, \theta)= & \frac{\Omega_{0}^{2}}{\left(1+\Omega_{0} \omega t\right)^{2}}\left(-\frac{1}{6}+3 \phi_{1} \cos ^{2} \theta-\phi_{1}\right) r^{2}, \\
P(t, r, \theta)= & \frac{P_{0}}{\left(1+\Omega_{0} \omega t\right)^{2 \gamma}}+\frac{\rho_{0} \Omega_{0}^{2}}{\left(1+\Omega_{0} \omega t\right)^{4}} \\
& \times\left(\Pi_{0}+\Pi_{1} \cos ^{2} \theta+\Pi_{2} \cos \theta \sin \theta\right) r^{2},
\end{aligned}
$$$$
V_{r}(t, r, \theta)=\frac{\Omega_{0}}{1+\Omega_{0} \omega t}
$$$$
\times\left(V_{0}-U_{1} \cos ^{2} \theta-U_{2} \cos \theta \sin \theta\right) r,
$$$$
V_{\theta}(t, r, \theta)=\frac{\Omega_{0}}{1+\Omega_{0} \omega t}\left(U_{1} \cos \theta \sin \theta+U_{2} \sin ^{2} \theta\right) r
$$$$
V_{\phi}(t, r, \theta)=\frac{\Omega_{0}}{1+\Omega_{0} \omega t} W_{1} \sin \theta r
$$$$
B_{r}(t, r, \theta)=\frac{\sqrt{\mu_{0} \rho_{0}} \Omega_{0}}{\left(1+\Omega_{0} \omega t\right)^{2}}
$$$$
\times K\left(-3 U_{1} \cos ^{2} \theta+U_{1}-3 U_{2} \cos \theta \sin \theta\right) r
$$

$$
\begin{aligned}
B_{\theta}(t, r, \theta) & =\frac{\sqrt{\mu_{0} \rho_{0}} \Omega_{0}}{\left(1+\Omega_{0} \omega t\right)^{2}} \\
\times & K\left(3 U_{1} \cos \theta \sin \theta+3 U_{2} \sin ^{2} \theta\right) r
\end{aligned}
$$

$B_{\phi}(t, r, \theta)=\frac{\sqrt{\mu_{0} \rho_{0}} \Omega_{0}}{\left(1+\Omega_{0} \omega t\right)^{2}} 3 Q \sin \theta r$.

With $U_{2}=\Pi_{2}=0\left(\gamma, \omega, \Pi_{0}, \Pi_{1}, U_{1}, V_{0}, W_{1}, Q\right.$ and $\phi_{1}$ being given by Eqs. (47), (48), (B.1), (B.2), (51), (B.3), (B.4), (63), (B.5), (B.6)), B being a free parameter,
Eqs. (1)-(6) admit the solutions given by Eqs. (A.1)-(A.6) and (A.9) and:

$$
\begin{aligned}
B_{r}(t, r, \theta)= & \frac{B}{\left(1+\Omega_{0} \omega t\right)^{2 V_{0} / \omega}} \cos \theta \\
& +\frac{\sqrt{\mu_{0} \rho_{0}} \Omega_{0}}{\left(1+\Omega_{0} \omega t\right)^{2}} K\left(-3 U_{1} \cos ^{2} \theta+U_{1}\right) r,(A \\
B_{\theta}(t, r, \theta)= & -\frac{B}{\left(1+\Omega_{0} \omega t\right)^{2 V_{0} / \omega}} \sin \theta \\
& +\frac{\sqrt{\mu_{0} \rho_{0}} \Omega_{0}}{\left(1+\Omega_{0} \omega t\right)^{2}} K\left(3 U_{1} \cos \theta \sin \theta\right) r
\end{aligned}
$$

\section{Appendix B: Aspherical temperature field}

In this appendix we give for completeness the solutions of Eqs. (68) and (70) that are symmetrical with respect to the equatorial plan $\left(U_{2}=0, \Pi_{2}=0\right)$ and briefly discuss their main properties.

\section{B.1. Aspherical pressure}

Two solutions with $W_{1}=K=Q=0$ are obtained:

$$
\begin{aligned}
& \left\{\begin{array}{l}
\Pi_{0}=-\Pi_{1}, \Pi_{0}=\frac{3 \phi_{1} \gamma-5 \phi_{1}-1 / 3+\gamma / 4}{3-2 \gamma}, \\
\omega= \pm \sqrt{\frac{2 \phi_{1}-1 / 6}{(1-\gamma)(3-2 \gamma)}}, U_{1}=(4-3 \gamma) \omega, \\
V(\pi / 2)=V_{0}=(2-\gamma) \omega, V(0)=2(\gamma-1) \omega .
\end{array}\right. \\
& \left\{\begin{array}{l}
\Pi_{0}=0, \Pi_{1}=-1 / 2+(3 \gamma-2)(2-\gamma) \omega^{2} / 4, \\
\omega= \pm \sqrt{\frac{4 / 3+8 \phi_{1}}{\gamma(2-\gamma)}}, U_{1}=(3 \gamma-4) \omega / 2, \\
V(\pi / 2)=V_{0}=\gamma \omega / 2, V(0)=(2-\gamma) \omega .
\end{array}\right.
\end{aligned}
$$

These two solutions describe a collapse or an expansion with an aspherical thermal pressure (Fig. 2 displays the velocity field of the solution stated by Eqs. (45)-(B.1) for $\gamma=5 / 3, \gamma=4 / 3$ and $\gamma=1.2)$. The gravitational potential can be uniform $\left(\phi_{1}=0\right)$ or aspherical $\left(\phi_{1} \neq 0\right)$. In the limit $\phi_{1} \rightarrow 0$ and $\gamma \rightarrow 4 / 3$, one recovers the solution stated by Eq. (47).

For the first solution (Eqs. (B.1)), a solution exists only if $\phi_{1}<1 / 12$ and $1<\gamma<3 / 2$ or if $\phi_{1}>1 / 12$ and $\gamma<1$ or $\gamma>3 / 2$. If $\gamma<1$ and $\phi_{1}>1 / 12$, the solution presents outflows at the pole.

For the second solution (Eqs. (B.2)), one has $\phi_{1}>$ $-1 / 6$ and $0<\gamma<2$.

\section{B.2. Aspherical pressure and rotation}

Two solutions with $K=Q=0$ are obtained:

$\left\{\begin{array}{l}\Pi_{0}=-\Pi_{1}, \omega= \pm \sqrt{4 / 3+8 \phi_{1}-4 W_{1}^{2}+8 \Pi_{0}}, \\ \phi_{1}=1 / 12, \gamma=3 / 2, U_{1}=-\omega / 2 \\ V(\pi / 2)=V_{0}=\omega / 2, V(0)=\omega .\end{array}\right.$

$$
\left\{\begin{array}{l}
\Pi_{0}=0, \omega= \pm \sqrt{4 / 3+8 \phi_{1}-4 W_{1}^{2}} \\
\phi_{1}=1 / 12+\Pi_{1} / 2, \gamma=1, U_{1}=-\omega / 2 \\
V(\pi / 2)=V_{0}=\omega / 2, V(0)=\omega
\end{array}\right.
$$


The polytropic index of the first solution (Eqs. (B.3)) is equal to $3 / 2$ and the gravitational potential is strongly constrainted $\left(\phi_{1}=1 / 12\right)$. The solution exists only if $\Pi_{0}>$ $-1 / 4$ and $W_{1}<\sqrt{1 / 2+2 \Pi_{0}}$.

The polytropic index of the second solution (Eqs. (B.4)) is equal to 1 . If $\Pi_{1}=-1 / 6$, the gravitational potential is isotropic $\left(\phi_{1}=0\right)$. The solution exists only if $W_{1}<\sqrt{1 / 3+2 \phi_{1}}$.

\section{B.3. Aspherical pressure, rotation and magnetic field}

Two solutions with magnetic fields, rotation, aspherical pressure are obtained:

$$
\left\{\begin{array}{l}
\Pi_{0}=-\Pi_{1}, \\
\Pi_{0}=-1 / 4-Q^{2}+W_{1}^{2} / 2-(\gamma-1)(3 \gamma-5) \omega^{2} / 2, \\
\omega= \pm \sqrt{\frac{2 \phi_{1}-1 / 6}{(1-\gamma)(3-2 \gamma)}}, W_{1}=2 Q K \frac{4-3 \gamma}{3-2 \gamma}, \\
U_{1}=(4-3 \gamma) \omega \\
V(\pi / 2)=V_{0}=(2-\gamma) \omega, V(0)=2(\gamma-1) \omega .
\end{array}\right.
$$

$$
\left\{\begin{array}{l}
\Pi_{0}=0, \\
\Pi_{1}=-1 / 2-2 Q^{2}+W_{1}^{2}+(3 \gamma-2)(\gamma-2) \omega^{2} / 4, \\
\omega= \pm \sqrt{\frac{8 \phi_{1}+4 / 3-4 W_{1}^{2}+8 Q^{2}}{\gamma(2-\gamma)}}, W_{1}=Q K \frac{3 \gamma-4}{\gamma-2}, \\
U_{1}=(3 \gamma-4) \omega / 2, \\
V(\pi / 2)=V_{0}=\gamma \omega / 2, V(0)=(2-\gamma) \omega .
\end{array}\right.
$$

The first solution (Eqs. (B.5)) is defined for $\phi_{1}<1 / 12$ and for $1<\gamma<3 / 2$ or $\phi_{1}>1 / 12$ and $\gamma<1$ or $\gamma>3 / 2$. The second one (Eqs. (B.6)) is defined if $W_{1}^{2}<1 / 3+2 \phi_{1}+$ $2 Q^{2}$. For both solutions $\phi_{1}$ can be equal to zero (isotropic potential) and the rotation parameter, $W_{1}$, is proportional to $Q K$.
As for Eqs. (B.1), if $\gamma<1$ and $\phi_{1}>1 / 12$, the first solution presents an outflow at the pole.

\section{References}

Arthur, S., \& Falle, S. 1991, MNRAS, 251, 93

Basu, S. 1997, ApJ, 485, 240

Blottiau, P., Bouquet, S., \& Chièze, J. P. 1988, A\&A, 207, 24

Bouquet, S., Feix, M., \& Fijalkow, E. 1985, ApJ, 293, 494

Chandrasekhar, S. 1967, Stellar Structure (New York)

Chiueh, T., \& Chou, J. 1994, ApJ, 431, 380

Contopoulos, I., Ciolek, G., \& Konigl, A. 1998, ApJ, 504, 247

Galli, D., \& Shu, F. 1993, ApJ, 417, 220

Goldreich, P., \& Weber, S. 1980, ApJ, 238, 991

Habe, A., Uchida, Y., Ikeuchi, S., \& Pudritz, R. 1991, PASJ, 43, 703

Hunter, C. 1977, ApJ, 218, 834

Larson, R. 1969, MNRAS, 145, 271

Li, Z. 1998, ApJ, 493, 230

Li, Z. 1998, ApJ, 497, 850

Lin, C., Mestel, L., \& Shu, F. 1965, ApJ, 142, 1431

Low, B. C. 1992, ApJ, 390, 567

Mouschovias, T. 1978, in Protostars and Planets, ed. T. Gehrels (Tucson: University of Arizona Press)

Munier, A., \& Feix, M. 1983, ApJ, 267, 344

Nakamura, F., Matsumoto, T., Hanawa, T., \& Tomisaka, K. 1999, ApJ, 510, 274

Nelson, R., \& Langer, W. 1997, ApJ, 482, 796

Penston, M. 1966, Roy. Obs. Bull., 117, 299

Penston, M. 1969a, MNRAS, 144, 425

Penston, M. 1969b, MNRAS, 145, 457

Ringeval, C., \& Bouquet, S. 2000 A\&A, 355, 564

Shu, F. 1977, ApJ, 214, 488

Tomisaka, K. 1998, ApJ, 502, L163

Tomisaka, K. 2001, ApJ, submited [astro-ph/0105527]

Whitworth, A., \& Summers, D. 1985, MNRAS, 214, 1

Yahil, A. 1983, ApJ, 265, 1047 